\title{
The Implications of Cognitive Femtocell Based Spectrum Allocation Over Macrocell Networks
}

\author{
Joydev Ghosh • Sanjay Dhar Roy
}

\begin{abstract}
We propose an analytical framework of significant influence of deployment of femto base stations (FBSs) in a dual-tier network constituted of macro base stations (MBSs). As because of uncoordinated deployment, FBSs results in destructive interference to MBSs and viceversa. But, throughput efficiency of indoor (Femtocell) and outdoor (Macrocell) environment substantially increases due to an optimum reutilization of available spectrum. The quality of service (QoS) is further getting optimized in terms of throughput and network coverage because of incorporation of steerable beamforming. Finally, the effectiveness of the scheme is verified by extensive matlab simulation.
\end{abstract}

Keywords Cognitive-Femtocell Networks, Throughput Efficiency, Network Coverage Analysis, Steerable Beamforming.

\section{Introduction}

Radio waves also fade quickly at upper frequencies, creating it difficulties to provide service to indoor subscribers with outdoor macro base stations (MBSs). Here, femtocells are come into consideration to figure out the problems to resolve prominently in the next phase of development after 4G. Femtocell standardisation exercise started in the year of 2007 through 3GPP (3rd Generation Partnership Project) is still in progress. Initial products launched to the market in 2008 [1] [2]. The Femto Base Station (FBS) is a low-priced miniature base station which facilitates same radio access compatibility as a general MBS about User Equipments (UEs).

\author{
Joydev Ghosh $\bigotimes$ \\ Dept. of Electronics and Telecommunications Engineering, NHIT, Durgapur, W.B, India \\ e-mail: joydev.ghosh.ece@gmail.com \\ Sanjay Dhar Roy \\ Dept. of Electronics and Communications Engineering, NIT, Durgapur, W.B, India
}


The FBS are exploited independently by users in common place or enterprise premises in a way of plug and play. Femtocells are designed to furnish indoor cellular access utilizing licensed here as Home Base Station (HBS), a low-power and short-coverage device which is coupled to the operator's core network via internet. The femtocell access policy is assessed in two ways: open access, where any user belonging to the operator's network can connect to the HBS and closed access, where only a specified set of users can privately couple to the HBS. Open access system is suitable for public places while closed access system is perfectly suited for home and office scenarios. Closed-access systems are therefore defenceless to larger interference from indoor users that situated geographically in coverage of femtocell but are not coupled to its FBS. Users entangled to a macrocell in downlink (DL) will experience a "coverage hole" nearby the femtocell, both for co-channel and immediate adjacent channel operation. Coverage holes mention to zone in the neighbourhood of a HBS where interference from HBS signals obstructs macro users to receive the fancy service from macro network. The area of coverage hole expands with the distance between MBS and HBS. Hence, the circumstances become deficient at macrocell edges.

In this paper, we theoretically analyzed the coverage holes for indoor scenario when the constant power transmitted by HBS and also when power adjustment utilizes [20]. Furthermore, we probe coverage holes for different carrier dissociation between macro and femto carrier frequencies. MBSs are located in the center of the circle and have an isotropic antenna. Statistics are accumulated from the cell at center of the round grid. The serving MBS emits at its highest transmission power $\left(\mathrm{P}_{\max (\mathrm{MBS})}\right)$ of $43 \mathrm{dBm}$. In general, the primary common pilot channel $(\mathrm{P}-$ $\mathrm{CPICH}$ ) power in 3GPP terminology is $33 \mathrm{dBm}$ [3]. P-CPICH is the downlink primary common pilot signal radiated by the MBS in OFDMA based cognitive-femtocell networks with constant power. P-CPICH is a code domain power and used for primary identification of scrambling codes which uniquely recognize every part in the network. The subject matter of co-channel and adjacent channel deployments are the issues recently being topic of a lot of studies, but assuming other separation distances, lesser than $5 \mathrm{MHz}$, can be of interest for spectrum allocation. The coverage hole is getting bigger and interference to the adjacent channel is getting enhanced due to decrease in carrier separation. Adjacent Channel Leakage Power Ratio (ACLR) and Adjacent Channel Selectivity (ACS) are the two parameters in connection with adjacent channel interference. ACLR is entangled with filters and disfigurement at the transmitter whereas ACS takes for the features of the receiver. In contrast to Omni-directional transmissions, beam forming reduces interference, allowing more concurrent transmissions in the network. The intention of considering beamforming is to improve signal to interference plus noise ratio (SINR). Additionally, by focusing the transmission energy in a particular direction, beamforming induces a signal that is in order of the magnitude stronger than that of the signals in undesired direction. This technique is utilized here to enhance the coverage of a specific zone or data rate or channel capacity of the system.

In particular, the major contributions of this paper are highlighted below-

- The transmitting signal $\left(\mathrm{T}_{\mathrm{x}(\mathrm{MBS})}\right)$ from MBS weakened and worsen quicker once the signal reaches indoors. Femtocells furnish way out to the difficulties present in macrocell-based system. So that, FBS network coverage is one of the prime concerns in indoor environment to get good quality of service (QoS). Here, we calculate the coverage radius 
of femtocell considering different parameters in a three-storey building (British convention) in connection with above relevant problem.

- We develop a dual-tier network model where beamforming includes at base station (BS) which has the potential to steer (rotate) their respective coverage areas as per demand. Steerable beams furnish a remarkable range add on and considerable multipath and interference rejection. The fundamental of beamforming is to let the signals fall onto the array within the principal lobe of the beam pattern pass through and in the intervening period of time attenuate all other signals in the minor lobe area. Since contributions from all sources in the minor lobe area are attenuated by the low minor lobes, better outcomes are expected in the beam domain simulation, which has been verified in result section.

- We propose an analytical view of adjacent channel interference ratio (ACIR), coverage hole by means of primary common pilot channel (P-CPICH $\mathrm{E}_{\mathrm{c}} / \mathrm{I}_{\mathrm{o}}$ ) power. And throughput of indoor and outdoor environment is simulated to get the optimum impact of the networks.

- We present comprehensive numerical outcome to vindicate the developed simplified network model and to exhibit the effectiveness of our proposed scheme.

The remainder of this paper is organized as follows. In Section 2, we describe system model to define propose network. In Section 3, the assumptions considered for execution of exact scenario in the simulation model. In Section 4, we present and analyse the numerical results. Ultimately, we finish this work in Section 5 with conclusion.

\section{System Model}

We scrutiny a femtocell deployment in a big indoor area considering a three-storey building. A compass of $100 \times 100 \mathrm{~m} 2$ is assumed and within it 300 non-overlapping HBSs are implanted. The HBS is considered to have a highest transmission power $\left(\mathrm{P}_{\max (\mathrm{HBS})}\right)$ of $20 \mathrm{dBm}$ and an isotropic antenna. It is also considered that the coverage region of the HBS is round shaped. The macrocell network incorporates of a round grid of $19 \mathrm{MBSs}$ with a radius of $1 \mathrm{Km}$ each. We slice up the hexagonal cell into three orthogonal service areas $S_{\mathrm{i}}$.

Fig.1 Femtocells operate in the presence of the macrocell network, insulated by the exterior walls of the residence.

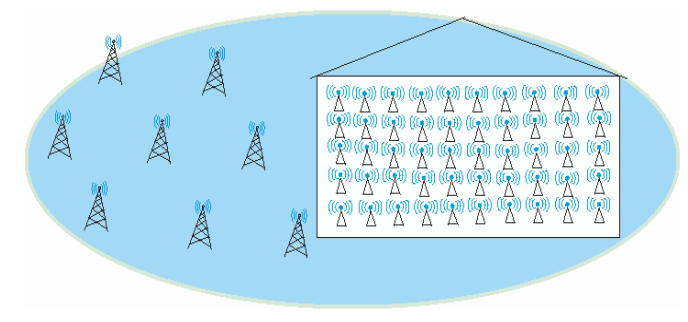

$i \in\{1,2,3\}$ to improve the throughput of macrocell networks. The beamformer constructs $\mathrm{M}_{\mathrm{t}}=3$ orthogonal steering beams $W_{i}=C^{N_{t} \times 1}$ for $\mathrm{i}=1, \ldots ., \mathrm{M}_{\mathrm{t}}$ in order to scrutinize all the service area. 
The unreasonable mobility needs limited feedback between the FAP and the UEs whereas co-tier interference needs a different radio resource cooperation scheme. Let $\mathrm{W}_{\mathrm{i}}=\left[\mathrm{w}_{\mathrm{i} 1}, \mathrm{w}_{\mathrm{i} 2}, \ldots, \mathrm{w}_{\mathrm{iMt}}\right]$ the orthogonal beamforming vector $\mathrm{M}_{\mathrm{t}} \times 1$ applied at the FAP/HBS with $\mathrm{M}_{\mathrm{t}}$ antenna elements. $\mathrm{w}_{i l}$ is the weight assigned at the antenna element 1 for transmitting at service area $i$. The beamforming vector applied at the $\mathrm{HBS} \mathrm{W}_{\mathrm{i}}(\mathrm{q})$ is fixed where $\mathrm{i}=\left\{1,2,3, \ldots, \mathrm{M}_{\mathrm{t}}\right\}$ throughout the q-th primary resource block (PRB) because of every chance of getting the experience of flat fading in the operation of narrowband PRBs in frequency and time domain. The transmitted signal can be written as:

$$
X_{i}(q)=W_{i}(q) S_{i}(q)
$$

where $S_{i}(q)=\left[S_{i 1}(q) S_{i 2}(q) S_{i 3}(q) \ldots . S_{i M_{t}}(q)\right]^{T}$ is the transmitted data vector, considering $E\left\{\left|S_{i}(m)\right|^{2}\right\}=1 . S_{i 1}(q)$ is complex data symbol transmitted from antenna element 1 in q-th PRB for the UEs which are located in area i. The HBS creates a set of up to $M_{t}=3$ at the starting point of each PRB, orthogonal beams $W_{i}(q)$ and necessarily the signal from the BS is the super position of these beams:

$$
X(q)=\sum_{i=1}^{M_{t}} X_{i}(q)=\sum_{i=1}^{M_{t}} W_{i}(q) S_{i}(q)
$$

The k-th mobile user estimates the downlink (DL) channel matrix $H_{k}(q)$ by the unique reference signals employing from cognitive-femtocell systems for channel estimation and designs the feedback that the HBS uses to embrace the transmitted signal to the channel. The DL channel matrix $H_{k=}\left[M_{r} \times M_{t}\right]$ can be re-expressed as $H_{k}(q)=\left[H_{k, r, t}\right]$ where $H_{k, r, t}$ represents the channel response between the receive antenna element \# $\mathrm{r}$ of k-th mobile user and the transmit antenna element \# t of HBS. The received signal $Y_{k}=C^{M_{r}}$ at the k-th UE can be re-expressed as:

$$
Y_{k}(q)=H_{k}(q) X(q)+n_{k}+I_{k, i}^{f}+I_{k, i}^{\prime f}
$$

where $\mathrm{n}_{\mathrm{k}}$ is the additive white gaussian noise (AWGN) with zero mean and variance $\sigma_{k}{ }^{2}, I_{k, i}^{f}$ and $I_{k, i}^{\prime f}$ is the cross-tier and co-tier interference, respectively, that the k-th UE experiences. The cochannel interference of $\left(M_{t}-1\right)$ interfering beams is eliminated because of orthogonality. The received signal is multiplied by a coefficient vector $U_{k}=\left[u_{k, 1}, u_{k, 2}, u_{k, 3}, \ldots \ldots u_{k, M_{t}}\right]^{T}$ and after that it is combined. We assume that channel state information (CSI) is known at the receiver prior to combine. The detector takes the coefficient vector $\mathrm{U}_{\mathrm{k}}$ in order to estimate the transmitted symbol

$$
\begin{aligned}
& \widehat{S_{k}}=u_{k}^{H} Y_{k}(\mathrm{q}) \\
& \widehat{S_{k}}=u_{k}^{H} h_{i k} S_{i}(q)+u_{k}^{H}\left(n_{k}+I_{k, i}^{f}+I_{k, i}^{\prime f}\right)
\end{aligned}
$$

where $h_{i k}=H_{k} W_{i}$ refers the projection of beam $W_{i}$ on the channel $H_{k}$. Then, the SINR computed at the receiver $k \in S_{i}$ is 


$$
\operatorname{SINR}_{i, k, n}=\frac{\left|u_{k}^{H} h_{i, k}\right|^{2}}{u_{k}^{2}\left(\sigma_{k}{ }^{2}+I_{k, i}^{f}+I_{k, i}^{\prime f}\right)}
$$

The achievable rate over the k-th UE can be written as:

$$
C_{i, k, n}=W \log _{2}\left(1+S I N R_{i, k, n}\right)
$$

where $W$ is allotment of band for $n$-th sub channel.

\subsection{Beamforming Pattern-}

Co-tier interference from adjacent HBS is a radical influence factor on the channel capacity which may also be affected by high mobility. Additional transmit power does not improve system performance much whereas there is a high chance of exceeding the limitation of specific absorption rate (SAR) and not only that it adds unnecessary co-tier interference. Total available spectrum is allocated to each service sector $\mathrm{S}_{\mathrm{i}}, i \in\{1,2,3\}$. The service sector $\mathrm{Si}$ is slice up into $\mathrm{N}_{\mathrm{B}}$ sub-areas of same size $\Delta \mathrm{S}$ which entangles with a grid of $\mathrm{N}_{\mathrm{B}}$ beams. A narrow width beam orthogonal in nature is steered to inspect the total area $S_{i}(\theta)=360^{\circ} / 3=120^{\circ}$. The phase is chosen to rotate the antenna array in a desire direction $\phi_{0}[4],[14]$. Keep one thing in mind that beamforming is let to remain unaltered in each PRB which is the scheduling interval. Cross-tier interference doesn't exist because three beams are orthogonal. And also, we incorporate the transmission of adjacent femtocell for the sake of combating inter-cell interference and hence, cell-edge performance improved. For instance, we incorporate the initial phase $\phi_{0 \mathrm{i}}$ of the overlapping region of three adjacent femtocell 1,2 , and $3, \phi_{0 \mathrm{i}}=\left\{0^{\circ}, 120^{\circ}, 240^{\circ}\right\}$ for femtocell 1 , femtocell 2, femtocell 3, respectively. The three narrow beams of femtocells 1 and femtocell 2 are steered in the clockwise direction wheras the beams at femtocell 3 in the anticlockwise direction. In this manner, the two steered beams which provide service to the cell-edge users of two non-overlapping adjacent femtocells which keep cross-tier interference $I_{k}$ at very minimum level.
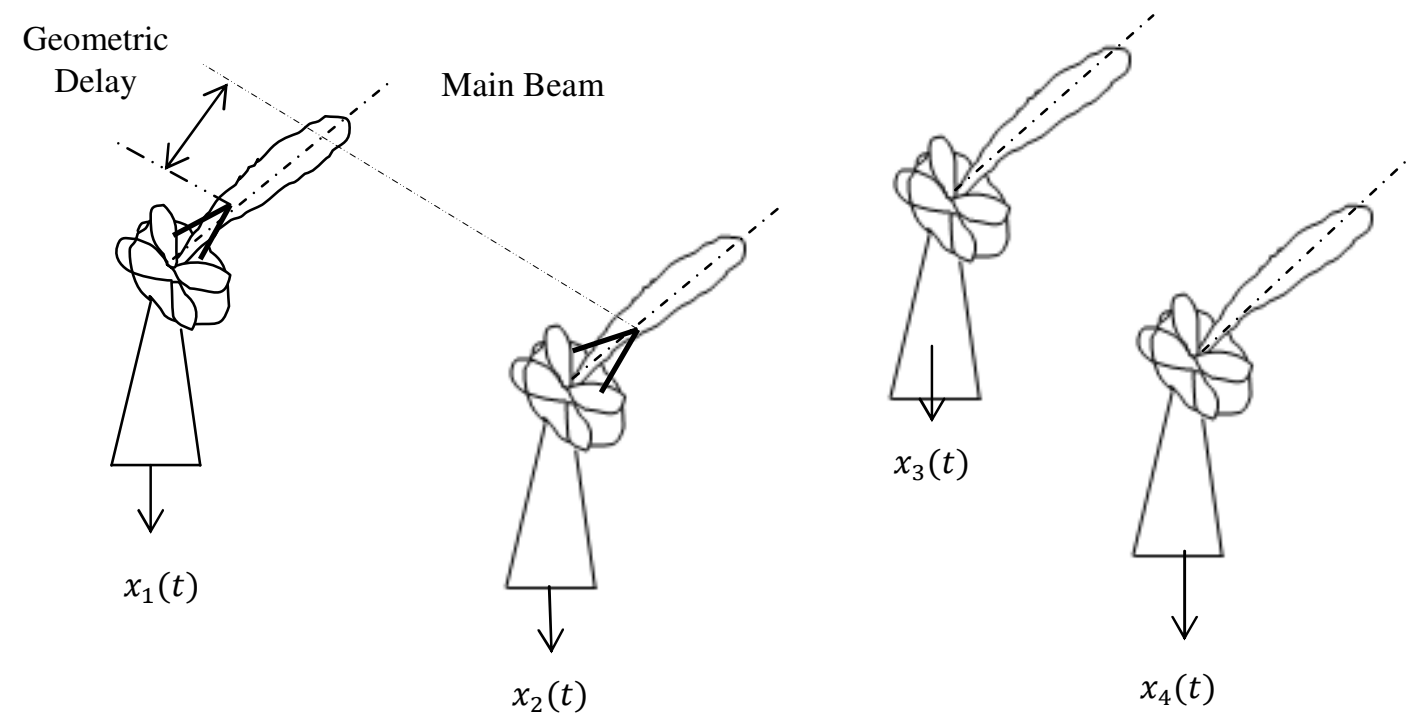
Fig. 2 Geometric location of discrete source model

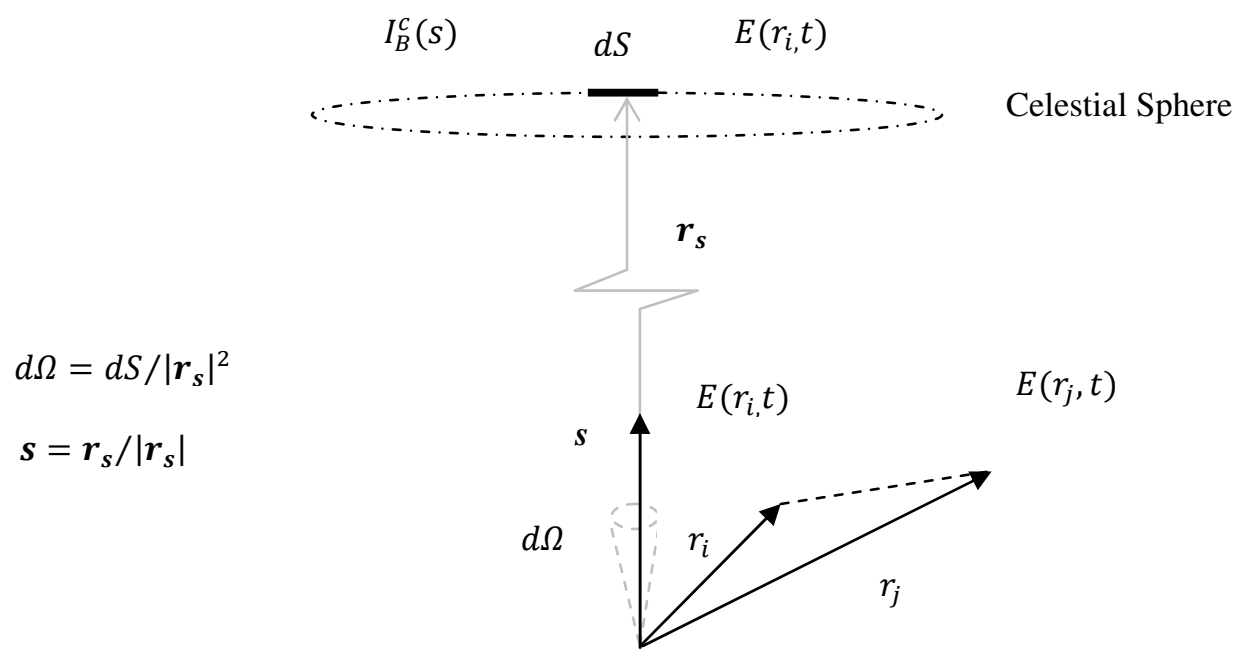

Fig.3 An interferometry sketch

Complex visibility function in terms of correlation function can be expressed as

$$
V\left(r_{i}, r_{j}\right)=E\left\{E\left(r_{i}, t\right), \overline{\left.E\left(r_{j} t\right)\right\}}=\int I_{B}^{c}(s) e^{-2 \pi j B c^{-1} s^{t}\left(r_{i}-r_{j}\right)} d \Omega\right.
$$

where B indicates bandwidth of $s(t), c^{-1}$ for interferers that are frequently moving, is providing penalty close to compensate losses in number of samples.

We Consider an array of $\mathrm{P}$ antennas with baselines of $b_{i j}=r_{i}-r_{j}$ where $r_{i}, r_{j}$ indicates sensor locations respectively. Signals $x_{i}(t)$ are staked in a vector whereas additive white gaussian noise signal (AWGN) is indicated by $n_{i}(t)$.

$$
\begin{aligned}
& X(t)=\left[x_{1}(t), x_{2}(t), \ldots . x_{p}(t)\right]^{t} \\
& n(t)=\left[n_{1}(t), n_{2}(t), \ldots . n_{p}(t)\right]^{t}
\end{aligned}
$$

Hence, $X(t)=a S(t)+n(t)$

where $a=\left[\gamma_{1} e^{-2 \pi j \frac{1}{\lambda} r_{1}^{t} s}, \ldots \ldots, \gamma_{p} e^{-2 \pi j \frac{1}{\lambda} r_{p}^{t} s}\right]^{t}$

Now sample estimate can be defined as [16], [17]$\hat{R}=\frac{1}{N} \sum_{n=1}^{N} x_{n} x_{n}^{H} \quad$ with $X_{n}=X\left(n T_{s}\right)$ for independent and identically distributed (i.i.d) noise vector $n(t)$ with $E\left\{n(t) n(t)^{H}\right\}=\sigma_{n}^{2} I$ where I is the identity matrix $E\left\{s(t)^{2}\right\}=\sigma^{2}$.

Hence, for single source, $R=E\{\hat{R}\}=\sigma^{2} a a^{H}+\sigma_{n}^{2} I$. 
And for multiple sources $\mathrm{R}$ can be re-expressed as, $R=\sum_{k} \sigma^{2} a a^{H}+\sigma_{n}^{2} I$

Now, random signals with $\mathrm{p}$ zero mean for given antenna element can be written as$X(t)=\left[x_{1}(t), x_{2}(t), \ldots x_{p}(t)\right]^{t}$, with $R=E\left\{X(t) X(t)^{H}\right\}$.

Let, beamformer output $y(t)=W^{H} X(t)$ where $W$ indicates beamformer weights.

Optimized beamformer output power,

$$
P=\max _{w} E|y(t)|^{2}=\max _{w}\left(w^{H} R w\right)
$$

where $w=\frac{a}{\sqrt{a^{H} a}}$

Therefore, beamformer output formula can be formulated by inserting the weight into it as follows-

$$
P(s)=\frac{a^{H} \hat{R} a}{a^{H} a}
$$

where the steering vector $a$ define as $a=e^{-2 \pi j R s \lambda^{-1}}$ with antenna location matrix,

$$
R=\left[b_{1}, b_{2}, \ldots, b_{p}\right]^{t}
$$

\subsection{Path Loss Model Recommended by ITU-R P.1238-}

The pathloss exponent mention below account an implied allowance for transmission through walls and through other obstacles responsible for other loss factors likely to be confronted within a single floor of a building.

The basic model has the following form [8]:

$$
L_{\text {total }}=20 \log _{10} f_{c}+10 n \log _{10} r+L_{f}\left(n_{f}\right)-28
$$

where,

$\mathrm{f}_{\mathrm{c}}=$ Frequency $(\mathrm{MHz})$.

$\mathrm{n}=$ Path loss exponent.

$\mathrm{r}=$ Separation distance $(\mathrm{m})$ between the base station and portable terminal (where, $\mathrm{r}>1 \mathrm{~m}$ )

$\mathrm{n}_{\mathrm{f}}=$ No of penetrated floors

$\mathrm{L}\left(\mathrm{n}_{\mathrm{f}}\right)=$ Floor penetration factor 
Table 1: Path loss exponent $\mathrm{n}$ for the ITU-R model

\begin{tabular}{|c|c|c|c|}
\hline \multirow{2}{*}{$\begin{array}{c}\text { Frequency } \\
\text { in GHz }\end{array}$} & \multicolumn{3}{|c|}{ Environment } \\
\cline { 2 - 4 } & Residential & Office & Commercial \\
\hline 0.9 & - & 3.3 & 2.0 \\
$1.2-1.3$ & - & 3.2 & 2.2 \\
$1.8-2.0$ & 2.8 & 3.0 & 2.2 \\
4.0 & - & 2.8 & 2.2 \\
60.0 & - & 2.2 & 1.7 \\
\hline
\end{tabular}

Table 2: Floor penetration factor, $\mathrm{L}\left(\mathrm{n}_{\mathrm{f}}\right)[\mathrm{dB}]$ for the ITU-R model

\begin{tabular}{|c|c|c|c|}
\hline \multirow{2}{*}{$\begin{array}{c}\text { Frequency } \\
\text { in GHz }\end{array}$} & \multicolumn{3}{|c|}{ Environment } \\
\cline { 2 - 4 } & Residential & Office & Commercial \\
\hline & & $9(1$ floor $)$ & \\
0.9 & - & $19(2$ floor $)$ & - \\
& & $24(3$ floor $)$ & \\
\multirow{2}{*}{$1.8-2.0$} & $4 n_{\mathrm{f}}$ & $15+4\left(\mathrm{n}_{\mathrm{f}}-1\right)$ & $6+3\left(\mathrm{n}_{\mathrm{f}}-1\right)$ \\
& & & \\
\hline
\end{tabular}

* Note that the penetration loss may be overestimated for large numbers of floors.

\subsection{Coverage Calculation of Femtocell Network-}

It is essential first to perceive the power levels which are necessitated to generate ample femtocell coverage in the non-appearance of co-channel interference. The utmost acceptable path loss necessitated to produce an enough pilot channel signal quality $\left(\frac{E_{c}}{N_{o}}\right)_{\text {femto }}$ while the femtocell is located faraway or in a distant location or may be in indoor place where MBS signal not available is then given by [8]:

$$
L_{\max }=10 \log \left(\frac{P_{\max }}{N_{U E}} \cdot\left(\frac{P_{C P I C H}}{\left(\frac{E_{c}}{N_{o}}\right)_{f e m t o}}-1\right)\right)
$$

where the utmost power transmitted by the FBS $(\mathrm{mW})$ is denoted by $\mathrm{P}_{\max }$, receiver noise power present in UE receiver is denoted by $\mathrm{N}_{\mathrm{UE}}$ and $\mathrm{P}_{\mathrm{CPICH}}$ represents amount of FBS power assigned to the pilot channel.

Equation no. (10) can be re-expressed as: 


$$
R=10 n \log _{10} r=L_{\text {total }}-20 \log _{10} f_{c}-L_{f}\left(n_{f}\right)+28
$$

Separation distance $(\mathrm{m})$ between the base station and movable terminal can be written as:

$$
r=10^{\frac{R}{10 n}} \quad \forall n=2
$$

The path loss $\mathrm{L}_{\text {total }}$ predicted by this model represents the median path loss at a distance $r$. To generalise it into the loss corresponding to a larger proportion of locations, the total loss has to be included as a summation of this loss and an extra shadow fade margin, $\mathrm{L}_{\mathrm{FM}}$. When the model is to be utilized to evaluate propagation between an indoor location and a nearest outdoor location, additionally a loss component of outer wall of the building added. Hence, the total loss is given by:

$$
L_{\text {total }}=L_{\max }-L_{F M}-L_{W}
$$

\subsection{Carrier Separation Analysis-}

The amount of adjacent channel interference is modeled by means of the Adjacent Channel Interference Ratio (ACIR) which is given by [15]:

$$
A C I R_{D L}=\frac{1}{\frac{1}{A C L R_{B S}}+\frac{1}{A C S_{M U E}}}
$$

where ACIR in the downlink (DL) of a macrocell user terminal (UE). ACLR $=45 \mathrm{~dB}$ and $\mathrm{ACS}=33 \mathrm{~dB}$ are the typical recent values used in [3]. The lesser these value the higher the adjacent channel interference. Remember that the arbitrary closely posture of Eq. (15) results to $\mathrm{CIR}=\min (\mathrm{ACLR}, \mathrm{ACS})$, hence for a carrier spacing of $5 \mathrm{MHz}, \mathrm{ACIR}=\mathrm{ACS}=33 \mathrm{~dB}$.

\subsection{Coverage Hole Calculation of Macrocell Network-}

MBS coverage holes are analyzed taking the deliberation of least needs for voice services. A guesstimate of this essential is that the pilot carrier to interference ratio (P-CPICH Ec/Io) should be larger or equal to $-18 \mathrm{~dB}[5],[15]$.

We assume an OFDMA based system for this dual-tier heterogeneous network (HetNets). In every iteration, we calculate the P-CPICH Ec/Io at a victim MUE and thereafter we consider the average over total samples. In the downlink, Ec/Io is achieved as [15], [18]:

$$
\frac{E_{c}}{I_{o}}=\frac{R S C P}{R S S I}=\frac{\frac{c_{k} P_{C_{C I C H_{k}}}}{L_{M_{k}}}}{\frac{c_{k} P_{T_{k}}}{L_{M_{k}}}+\sum_{\substack{i=1 \\ i \neq k}}^{N}\left(\frac{c_{i} P_{T_{i}}}{L_{M_{i}}}\right)+\sum_{j=1}^{H}\left(\frac{s_{j} P_{F B S_{j}}}{L_{F_{j}} A C I R}\right)+P_{B}}
$$


where RSCP denotes the received signal code power, which concur with the pilot signal P$\mathrm{CPICH}$ ( $\left.\mathrm{RSCP}_{\mathrm{P}-\mathrm{CPICH}}\right)$. RSSI represents the received signal strength indicator and it is the total power observed at the receiving end which adds the power observed by the serving network entity (MBS), the interference power from nearby MBSs, the interference power from nearby FBSs and the noise power at the UE. ACIR is a quantity of adjacent channel interference. The transmit powers of the serving base station (macrocell) is $\mathrm{P}_{\mathrm{Tk}}$ wheras transmit power of the nearby BSs (Macrocells) is $\mathrm{P}_{\mathrm{Ti}} . \mathrm{P}_{\mathrm{FBSj}}$ is the summation of total transmitted power by the serving FBS and nearby FBSs; $C_{i}$ denotes log-normal shadow fading for indoor and $\mathrm{s}_{\mathrm{j}}$ for outdoor scenario. The number of nearby MBSs and FBSs are denoted by $N$ and $H$, respectively. Eventually, $L_{M i}$ and $L_{F j}$ are pathloss models for the macrocell networks and femto networks, respectively, for a radio frequency operation of $2 \mathrm{GHz}$ range (Table 1). The channel to interference ratio (CIR) for a voice data channel of a FUE is modeled as given below [6], [15], [18]:

$$
C I R=\frac{\frac{0.75 s_{l} P_{F B S}}{L_{F_{l}}}}{\frac{C_{k} P_{T_{k}}}{L_{H} A C I R}+\sum_{\substack{i=1 \\ i \neq k}}^{N} \frac{C_{i} P_{T_{i}}}{L_{M_{i}} A C I R}+\sum_{j=1}^{H} \frac{S_{j} P_{F B S_{j}}}{L_{F_{j}}}+P_{B}}
$$

$0.1 \mathrm{P}_{\mathrm{FBS}}$ represents the proportion of power allocated for the intelligent signal associated with FBS to evaluate the channel to interference ratio.

\subsection{FBS Power Adjustment-}

We analyze a mechanism for power adjustment in the FBS based on the macrocell $\mathrm{RSCP}_{\mathrm{P}-\mathrm{CPICH}}$ quantifications of adjacent channels. Hence, P-CPICH signals are obtainable all the time, it is practicable to evaluate it though the adjacent channel be the property of a different operator. Besides, evaluation of $\mathrm{RSCP}_{\mathrm{P}-\mathrm{CPICH}}$ is ease by the fact that it is transmitted at $33 \mathrm{dBm}$ constant power which converts it into an easy computation at the FBS. The $\mathrm{RSCP}_{\mathrm{P}-\mathrm{CPICH}}$ is mean over iteration of 100 transmissions slots and next the output power control at the FBS is run as follows [15], [18]:

$P_{t_{H B S}}=\min \left(\max \left(P_{F B S_{\text {min }}}, R S C P_{P-C P I C H}+L_{f}+A C I R-E_{c}\left(I_{\text {oTarget }}\right), P_{F B S_{\text {max }}}\right)\right)$

where typical value of $\mathrm{P}_{\mathrm{FBSmin}}, \mathrm{P}_{\mathrm{FBSmax}}, \mathrm{Ec} / \mathrm{I}_{\mathrm{oTarget}}, \mathrm{ACIR}$ and $\mathrm{L}_{\mathrm{F}}$ are $0 \mathrm{dBm}, 20 \mathrm{dBm},-18 \mathrm{~dB}, 33$ $\mathrm{dB}$ and $47 \mathrm{~dB}$, respectively [7]. The computation of the macrocell RSCP is influenced by the forbearance of the sensor at the UE. For example a portable terminal may have a forbearance of about $7 \mathrm{~dB}$ in its macrocell RSCP quantifications [7]. We assume the case of perfect quantification and the case when the quantification error is log-normally distributed with standard deviation $=7 \mathrm{~dB}$.

\section{Simulation Model}

The simulation is developed in MATLAB with the use of ITU-R model in Table $1 \& 2$. In order to vindicate the excellence predicted by the analytical framework discussed in the previous 
section, we simulate different parameters at different conditions. Particularly, each simulation execution is carried out according to the following steps.

\subsection{Implantation of Macrocell (Outdoor) and Femtocell (Indoor)}

The following steps are followed to implant macrocell and femtocell successfully under indoor and outdoor scenario.

1. Each powerful MBS is located at the center of macrocell which is having the coverage of $1 \mathrm{Km}$ radius. The outdoor network incorporates of circular gride of 19 MBS. The transmitting signal $\left(\mathrm{T}_{\mathrm{x}(\mathrm{MBS})}\right)$ from MBS attenuated while the signal reaches indoors. The network performance is under serious measure especially due to poor coverage and underutilize spectrum in border area.

2. The coverage of $200 \mathrm{~m}$ radius femto base stations (FBSs) are densely deployed in that area where macro base station (MBS) unable to provide QoS and spectrum also not been utilized efficiently. The location of implantation of low power FBS is strictly inside the indoor area. The indoor network incorporates of circular gride of 300 FBS.

\subsection{Generation of Users' Location, Beamforming and Interference}

The generation of the users' locations and interference power is carried out considering the following steps.

1. A fixed number of outdoor users' $\left(\mathrm{N}_{\mathrm{MUE}, \mathrm{ku}}\right)$ and indoor users' $\left(\mathrm{N}_{\mathrm{FUE}, \mathrm{ji}}\right)$ is generated and they are randomly distributed within their own coverage area . $\mathrm{N}_{\mathrm{UE}}$ includes all MBSUs /PUs ( $\mathrm{N}_{\mathrm{MUE}, \mathrm{ku}}$ ) and FBSUs/SUs $\left(\mathrm{N}_{\mathrm{FUE}, \mathrm{ji}}\right)$ which means $\mathrm{N}_{\mathrm{UE}}=\mathrm{N}_{\mathrm{MUE}, \mathrm{ku}}+\mathrm{N}_{\mathrm{FUE}, \mathrm{ji}}$. Here, $j \in \mathrm{N}_{\mathrm{F}}=\left\{1,2, \ldots \ldots ., \mathrm{N}_{\mathrm{F}}\right\} ; k \in$ $\mathrm{N}_{\mathrm{M}}=\left\{1,2, \ldots \ldots, \mathrm{N}_{\mathrm{M}}\right\} ; \mathrm{N}_{\mathrm{MUE}, \mathrm{ku}} \forall\left\{1,2,3, \ldots \ldots, \mathrm{uN} \mathrm{N}_{\mathrm{M}}\right\}, \mathrm{u} \in$ any large integer value; $\mathrm{N}_{\mathrm{FUE}, \mathrm{ji}} \forall$ $\left\{1,2,3, \ldots, \mathrm{iN}_{\mathrm{F}}\right\}, \mathrm{i} \in$ any large integer value .

2. Beamforming gain is produced for each user can be obtained from Eq. (9) and the interference over the n-th sub-channel for cross tier and co-tier can be expressed as below [14]:

$$
\begin{aligned}
I_{k, i}^{f} & =\sum_{\mathrm{l}=1}^{\mathrm{N}_{\mathrm{M}}} P_{i, l, n}^{m} A_{i, l, n}^{m} G_{i, l, n}^{m} S_{i, l, n}^{m} \quad \forall l \in\left\{1,2,3 \ldots \ldots \mathrm{N}_{\mathrm{M}}\right\} \\
I_{k, i}^{f} & =\sum_{\mathrm{j}=1, \mathrm{j} \neq \mathrm{i}}^{\mathrm{N}_{\mathrm{M}} \mathrm{N}_{\mathrm{F}}} \beta_{j}^{n} P_{i, j, n}^{f} A_{i, j, n}^{f} G_{i, j, n}^{f} S_{i, j, n}^{f} \forall j \in\left\{1,2,3 \ldots \ldots \mathrm{N}_{\mathrm{M}}\right\}
\end{aligned}
$$

where $P_{i, l, n}^{m}$ and $P_{i, j, n}^{f}$ indicate the transmit signal powers over the $n$-th sub-channel of MBS $l$ and FBS j, respectively; $A_{i, l, n}^{m}$ and $A_{i, j, n}^{f}$ indicate the corresponding overall antenna gains for MBS $l$ and FBS j; $G_{i, l, n}^{m}$ and $G_{i, j, n}^{f}$ indicate the path gains, respectively; $S_{i, l, n}^{m}$ and $S_{i, j, n}^{f}$ indicate the 
shadowing gain, respectively. Uniform linear array (ULA) consider to measure the effectiveness of adaptive beamformer. Signal direction are collected from $-60^{\circ}, 0^{\circ}$ and $60^{\circ}$.

3. The received signal strength (RSS) is evaluated from PU/MBSU or SU/FBSU at the reference MBS or FBS.

4. Next, the SINR for a PU/Macro user and/or a SU/Femto user are computed.

\subsection{FBS Coverage, Throughput in Indoor and Outdoor Scenario Simulation}

The following steps are executed as follows-

1. To get the facility of good quality of service (QoS) network, how far FBSU can move from FBS is possible to predict from the simulation of coverage radius of FBS network. Simulation part of coverage radius in against of femtocell maximum transmit power of indoor network runs for different values of $\mathrm{P}_{\mathrm{CPICH}}, \mathrm{L}_{\mathrm{FM}}, \mathrm{N}_{\mathrm{UE}}, \mathrm{E}_{\mathrm{CN}}$.

2. Variation in throughput is computed based on indoor and outdoor scenario.

\section{Results and discussions}

In this section, we demonstrate interpretative numerical results to validate the effectiveness of the proposed network model. The network parameters and location of MBS and FBS for our simulations are shown in Fig. 1, where MBS users' and FBS users' are randomly located inside the coverage radius of $1000 \mathrm{~m}$ and $200 \mathrm{~m}$, respectively.

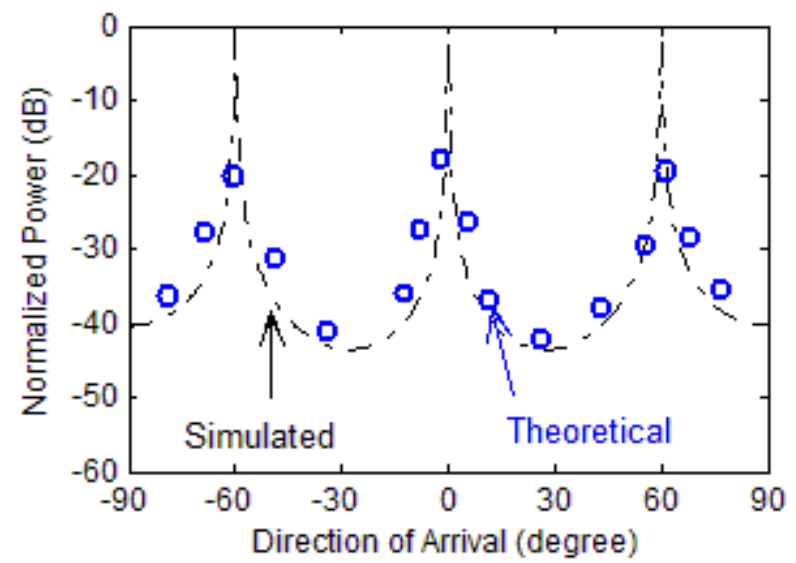

Fig.4 Normalized power vs. DOA for SINR=0

Fig 4 shows the adaptive beamformer spectrum generated with all array elements in presence of interference and noise. The output of an adaptive beamformer quantifies the quadratic measure of normalized power spectrum. Peaks of the DOA power spectrum gives DOA estimation. The results of DOA estimation are then added in ULA to design the adaptive beamformer, which is utilized to optimize the power radiated towards femto user (FU)/macro user (MU), and to attenuate interference.

In Fig.5, coverage radius of femtocell network is shown as a function of maximum transmit power $(\mathrm{dBm})$ of FBS where primary common pilot channel $(\mathrm{P}-\mathrm{CPICH})$ power chosen 0.1 and 0.2 with $1^{\text {st }}$ floor separation, $2^{\text {nd }}$ floor separation, $3^{\text {rd }}$ floor separation in a three storey building. 


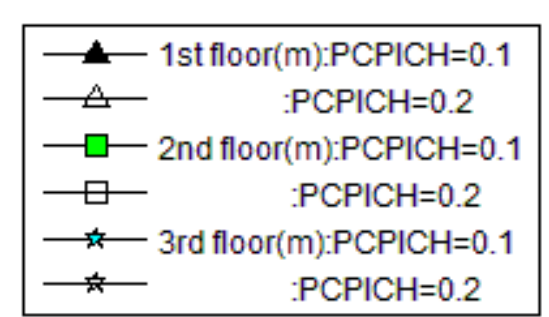

$\mathrm{PCPICH}=0.2$

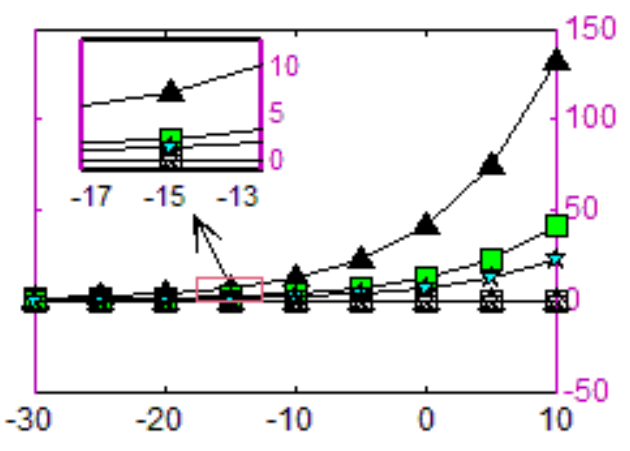

Maximum Transmit Power of FBS (dBm)

Fig.5 Coverage radius of FBS in indoor environments, Considering $\left(\frac{E_{c}}{N_{o}}\right)_{\text {Femtocell }}=-16 d B, \mathrm{~N}_{\mathrm{UE}}=5 \mathrm{~dB}$, $\mathrm{L}_{\mathrm{FM}}=9.9 \mathrm{~dB}, \mathrm{~L}_{\mathrm{w}}=10 \mathrm{~dB}$, also taking indoor location at the cell edge.

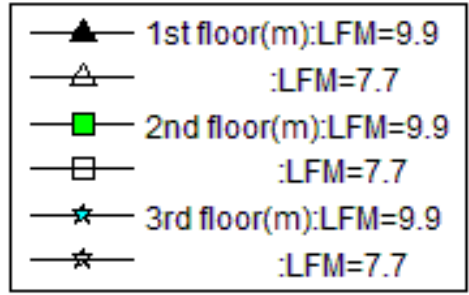

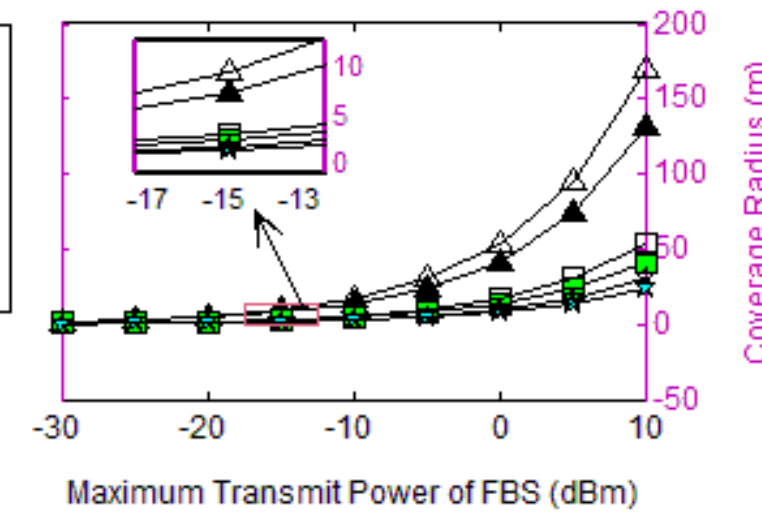

Fig.6 Coverage radius of FBS in indoor environments, Considering $\left(\frac{E_{C}}{N_{o}}\right)_{\text {Femtocell }}=-16 d B, \mathrm{~N}_{\mathrm{UE}}=5 \mathrm{~dB}$, $\mathrm{P}_{\mathrm{CPICH}}=0.1 \mathrm{~dB}, \mathrm{~L}_{\mathrm{w}}=10 \mathrm{~dB}$, also taking indoor location at cell edges.

In Fig.6, coverage radius of femtocell network is shown as a function of maximum transmit power $(\mathrm{dBm})$ of FBS where shadow fade margin $\left(\mathrm{L}_{\mathrm{FM}}\right)$ chosen as $7.7 \mathrm{~dB}$ and $9.9 \mathrm{~dB}$ with $1^{\text {st }}$ floor separation, $2^{\text {nd }}$ floor separation, $3^{\text {rd }}$ floor separation in a three storey building.

In Fig.7, coverage radius of femtocell network is shown as a function of maximum transmit power $(\mathrm{dBm})$ of FBS where user equipment received noise power $\left(\mathrm{N}_{\mathrm{UE}}\right)$ chosen as $5 \mathrm{~dB}$ and $7 \mathrm{~dB}$ with $1^{\text {st }}$ floor separation, $2^{\text {nd }}$ floor separation, $3^{\text {rd }}$ floor separation in a three storey building.

In Fig.8, coverage radius of femtocell network is shown as a function of maximum transmit power $(\mathrm{dBm})$ of FBS where pilot channel signal quality $\left(\left(\frac{E_{c}}{N_{o}}\right)_{\text {Femtocell }}\right)$ chosen as $5 \mathrm{~dB}$ and $7 \mathrm{~dB}$ with $1^{\text {st }}$ floor separation, $2^{\text {nd }}$ floor separation, $3^{\text {rd }}$ floor separation in a three storey building. 


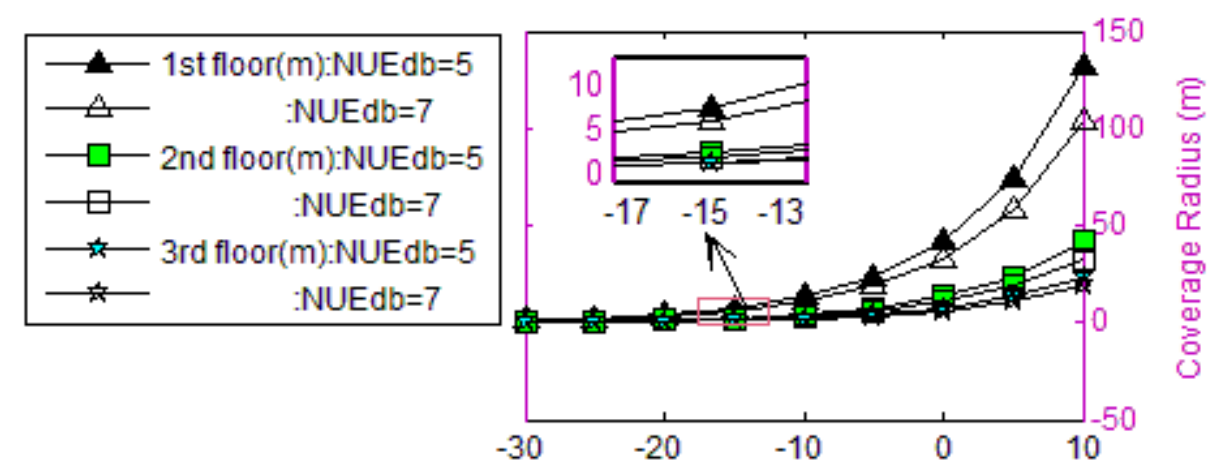

Femtocell Maximum Transmit Power (dBm)

Fig.7 Coverage radius of FBS in indoor environments, Considering $\left(\frac{E_{c}}{N_{o}}\right)_{\text {Femtocell }}=-16 d B$, $\mathrm{L}_{\mathrm{FM}}=9.9 \mathrm{~dB}$, $\mathrm{P}_{\mathrm{CPICH}}=0.1 \mathrm{~dB}, \mathrm{~L}_{\mathrm{w}}=10 \mathrm{~dB}$, also taking indoor location at cell edges.

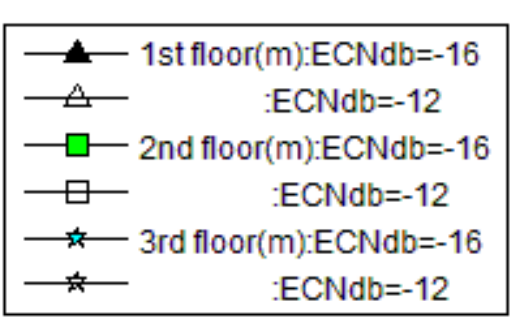

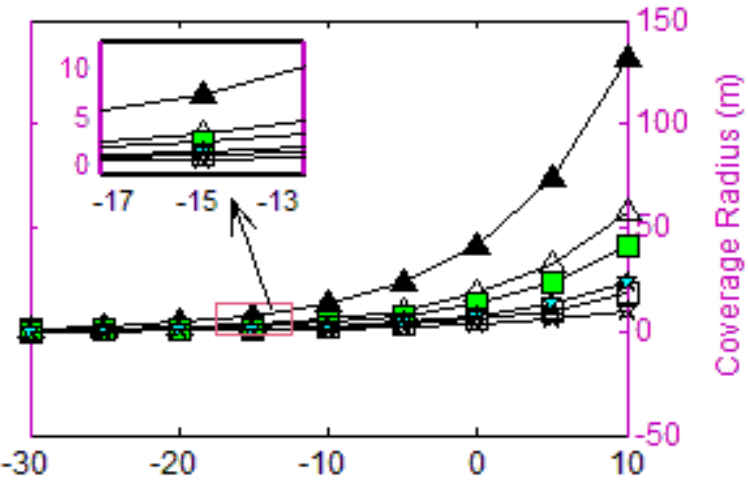

Femtocell Maximum Transmit Power (dBm)

Fig. 8 Coverage radius of FBS in indoor environments, Considering $\mathrm{N}_{\mathrm{UE}}=5 \mathrm{~dB}, \mathrm{~L}_{\mathrm{FM}}=9.9 \mathrm{~dB}, \mathrm{P}_{\mathrm{CPICH}}=0.1 \mathrm{~dB}, \mathrm{~L}_{\mathrm{w}}=10$ $\mathrm{dB}$, also taking indoor location at cell edges.

Figs. 5, 6, 7 and 8 illustrate the overall limitation of mobile users' to move how far from its generic network under the indoor scenarios where it is also considered that three storey building located at cell edges.

In Fig.9, we illustrate throughput with incorporation of beamforming in indoor scenario where beamformer is basically function of no. of antenna elements keeping FBS power constant. Beam is becoming more directive with increase in number of array elements which results in very high directive gain at a particular direction.

In Fig.10, throughput of macrocell networks is shown as a function of no. of femtocells in which different FBS transmit power set for simulation.

In Fig.11, throughput of macrocell networks is shown as a function of no. of femtocells in which different FBS transmit power set for simulation. 


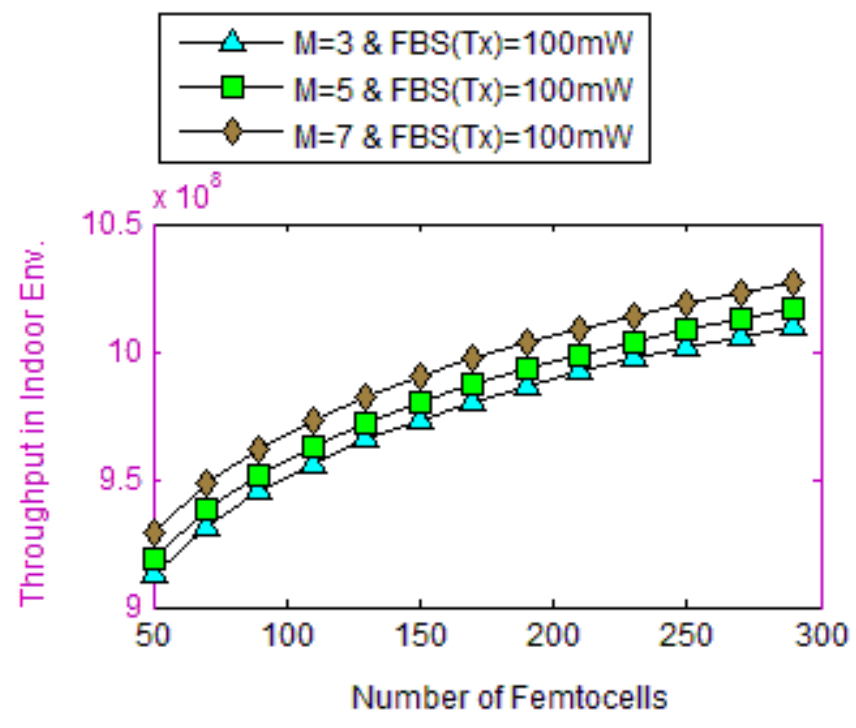

Fig.9 Throughput under the influence of beamforming in association with femtocells in indoor environments, considering $\left(\frac{E_{c}}{N_{o}}\right)_{\text {Femtocell }}=-16 \mathrm{~dB}, \mathrm{~N}_{\mathrm{UE}}=5 \mathrm{~dB}, \mathrm{~L}_{\mathrm{FM}}=9.9 \mathrm{~dB}, \mathrm{P}_{\mathrm{CPICH}}=0.1 \mathrm{~dB}, \mathrm{~L}_{\mathrm{w}}=10 \mathrm{~dB}$, also taking indoor location at cell edges.

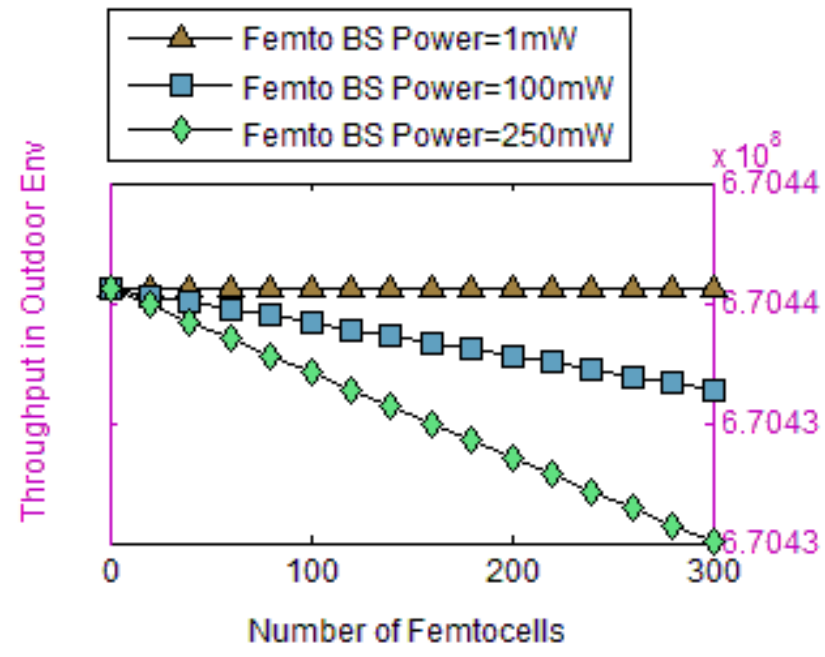

Fig.10 Throughput under the influence of transmitting power from associated femtocells in outdoor environments, considering $\left(\frac{E_{c}}{N_{o}}\right)_{\text {Femtocell }}=-16 \mathrm{~dB}, \mathrm{~N}_{\mathrm{UE}}=5 \mathrm{~dB}, \mathrm{~L}_{\mathrm{FM}}=9.9 \mathrm{~dB}, \mathrm{P}_{\mathrm{CPICH}}=0.1 \mathrm{~dB}, \mathrm{~L}_{\mathrm{w}}=10 \mathrm{~dB}$, also taking indoor location at cell edges.

In Fig.12, throughput of indoor networks is shown as a function of no. of femtocells in which different FBS transmit power set for simulation which signifies that throughput increases with the increase in FBS transmit power. 


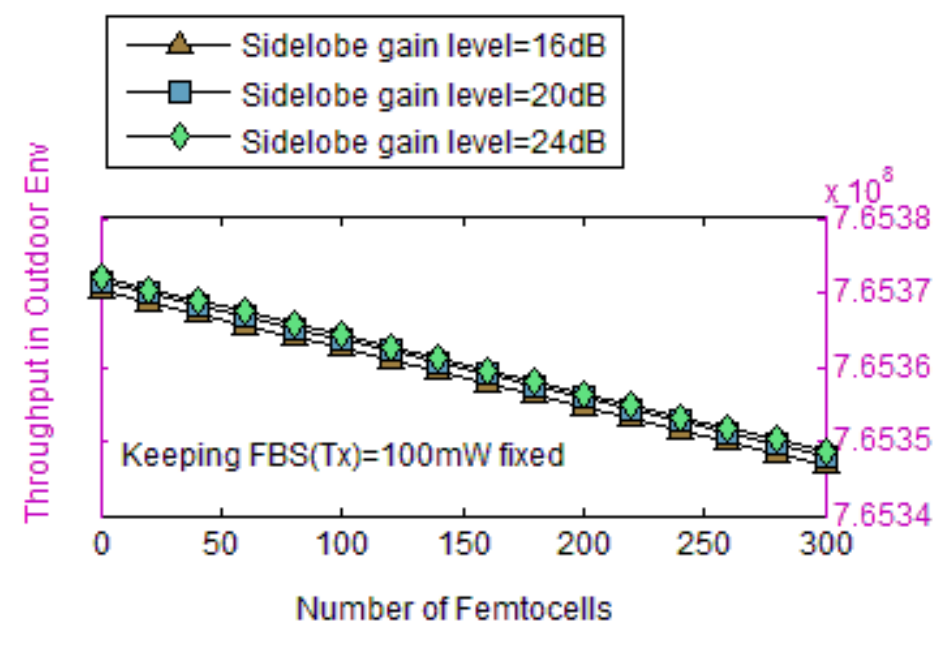

Fig.11 Throughput under the influence of side lobe gain in association with femtocells in outdoor environments, considering $\left(\frac{E_{C}}{N_{o}}\right)_{\text {Femtocell }}=-16 \mathrm{~dB}, \mathrm{~N}_{\mathrm{UE}}=5 \mathrm{~dB}, \mathrm{~L}_{\mathrm{FM}}=9.9 \mathrm{~dB}, \mathrm{P}_{\mathrm{CPICH}}=0.1 \mathrm{~dB}, \mathrm{~L}_{\mathrm{w}}=10 \mathrm{~dB}$, also taking indoor location at cell edges.

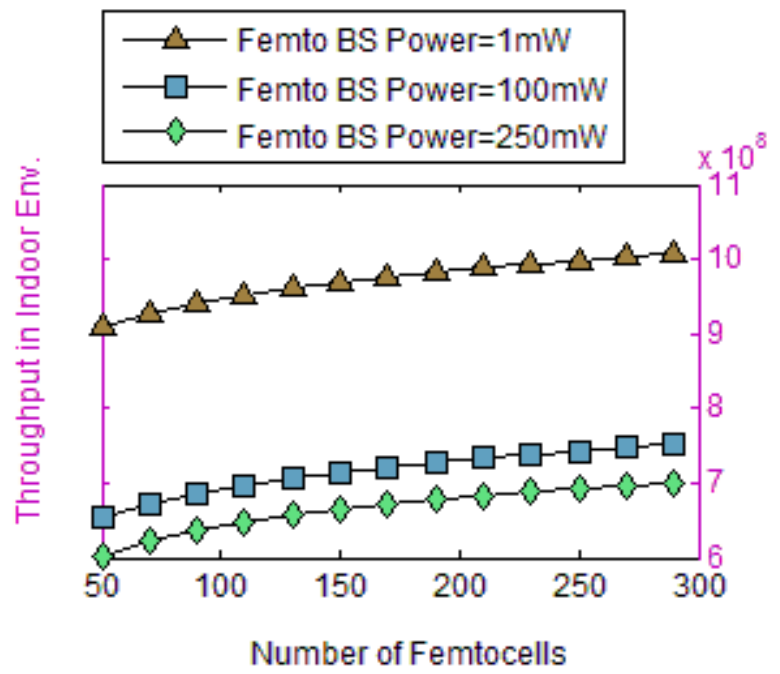

Fig.12 Throughput under the influence of side lobe gain in association with FBS in indoor environments, considering $\left(\frac{E_{c}}{N_{o}}\right)_{\text {Femtocell }}=-16 \mathrm{~dB}, \mathrm{~N}_{\mathrm{UE}}=5 \mathrm{~dB}, \mathrm{~L}_{\mathrm{FM}}=9.9 \mathrm{~dB}, \mathrm{P}_{\mathrm{CPICH}}=0.1 \mathrm{~dB}, \mathrm{~L}_{\mathrm{w}}=10 \mathrm{~dB}$, also taking indoor location at cell edges.

In Fig.13, we illustrate throughput of indoor networks as a function of no. of femtocells in which keeping FBS transmit power fixed where it is shown that throughput decreases with the increase in side lobe gain level due to loss of considerable amount of energy. 


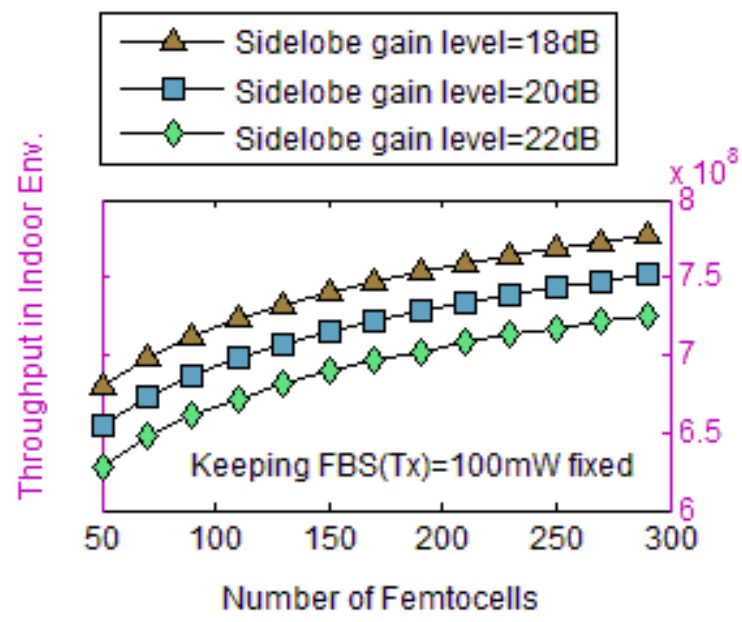

Fig.13 Throughput under the influence of side lobe gain in association with FBS in indoor environments, considering $\left(\frac{E_{c}}{N_{o}}\right)_{\text {Femtocell }}=-16 \mathrm{~dB}, \mathrm{~N}_{\mathrm{UE}}=5 \mathrm{~dB}, \mathrm{~L}_{\mathrm{FM}}=9.9 \mathrm{~dB}, \mathrm{P}_{\mathrm{CPICH}}=0.1 \mathrm{~dB}, \mathrm{~L}_{\mathrm{w}}=10 \mathrm{~dB}$, also taking indoor location at cell edges.

\section{Conclusions}

In this paper, we develop a novel simulation testbed model to demonstrate various aspects in terms of substantial parameters in connection with network coverage and throughput of indoor and outdoor scenario for dual-tier macrocell-femtocell networks. Femtocell network coverage for a three-storey building and coverage hole of macrocell network have been analyzed in this paper. Beamforming mechanism integrated at serving network entity (FBS) aims to reduce user effective interference. There is appreciable impact on throughput for number of antenna elements at FBS in beamforming mechanism. FBS coverage radius is focused in this particular research work to improve network performance by avoiding outage user which are generally located at cell edges.

\section{Reference}

[1] G. Mansfield, "Femtocells in the US Market -Business Drivers and Consumer Propositions," Femtocells Europe, ATT, London, U.K., June 2008. Femto Forum, www.femtoforum.org.

[2] Douglas N. Knisely, Airvana, Inc. Takahito Yoshizawa, Thomson Telecom Frank Favichia, AlcatelLucent,"Standardization of Femtocells in 3GPP," IEEE Communications Magazine, September 2009.

[3] 3GPP, "Universal Mobile Telecommunications System (UMTS); Base Station (BS) radio transmission and reception (FDD) 3GPP TS 25.104 version 11.3.0 Release 11“, 3GPP (2012-10). http://www.etsi.org.

[4] Godara. L. C. ,"Application of antenna arrays to mobile communications. Part II: Beam-forming and direction of arrival considerations," Proceedings of the IEEE, Vol. 85, No. 8, Aug.1997.

[5] Ericsson, "Downlink co-existence between macro cells and adjacent channel Home NodeBs.“, 3GPP TSGRAN WG4, R4-080939, Meeting \# 47, Kansas City, USA, 5th - 9th May, 2008.

[6] Ericsson, "Simulation assumptions for the block of flats scenario.“, 3GPP TSG-RAN WG4, R4-080149, Meeting \# 46, Sorrento, Italy, $11^{\text {th }}-15$ th February, 2008. 
[7] 3GPP, "Universal Mobile Telecommunications System (UMTS); Base Station (BS) radio transmission and reception (FDD) 3GPP TS 25.104 version 8.5.0 Release 8“, 3GPP 2009.

[8] Simon R. Saunders, Stuart Carlaw, Andrea Giustina, Ravi Raj Bhat, V. Srinivasa Rao and Rasa Siegberg," Femtocells: Opportunities and Challenges for Business and Technology," John Wiley \& Sons Ltd, 2009, ISBN: 9780-470-74816-9.

[9]V. Chandrasekhar, J. G. Andrews, and A. Gatherer,'Femtocell networks: A survey," IEEE Communication.Mag., vol.46, no. 9, pp.59-67, Sep.2008.

[10]FCC spectrum Policy Task Force. Report of the spectrum Efficiency Working Group, Tech. Rep. ET Docket No. 02-135, Washington DC (USA): Federal Communication Commission, 2002.

[11]Mitola J., Maguire G."Cognitive radio: Making software radios more personal," IEEE Personal Communications, 1999, vol. 6, no. 4, p.13-18.

[12] IEEE SCC34/SC2. IEEE recommended practice for determining the spatial-peak specific absorption rate (SAR) in the human body due to wireless communications devices: experimental techniques. 2000.

[13] Meredith Sharples, Vodafone UK. Femtocell business models for UK operators. Informa, 2008.

[14] Sanjay Dhar Roy, and Sumit Kundu,'Performance Analysis of Cellular CDMA in presence of Beamforming and Soft Handoff," Progress in Electromagnetics Research, PIER 88, pp-73-89, 2008.

[15] Espino J., Markendal J.,"Analysis of macro-femtocell interference and implications for spectrum allocation," IEEE International Symposium on Personal, Indoor and Mobile Radio Communications, 2009.

[16] J. van der Veen, A. Leshem, and A.J. Boonstra, "Array Signal Processing for Radio Astronomy", Experimental Astronomy (2004) 17: 231-240.

[17] Alle-Jan van der Veen1, Amir Leshem2 and Albert-Jan Boonstra3,"Signal Processing for Radio Astronomical Arrays", IEEE SAM,2004.

[18] Espino, J., Markendahl, J., "Analysis of macro - femtocell interference and implications for spectrum allocation", IEEE 20th International Symposium on Personal, Indoor and Mobile Radio Communications, 2009.

[19] Afaz Uddin Ahmed , Mohammad Tariqul Islam , Mahamod Ismail," A Review on Femtocell and its Diverse Interference Mitigation Techniques in Heterogeneous Network", Wireless Personal Communications (WPC), 2014, vol.78, no.1.

[20] Oh, D.-C., Lee, H.-C., \& Lee, Y.-H,”Cognitive radio based femtocell resource allocation,” International Conference on Information and Communication Technology Convergence (ICTC), 2010, 274-279.

[21] Srinivas Nallagonda, Sanjay Dhar Roy and Sumit Kundu, "Performance Evaluation of Cooperative Spectrum Sensing Scheme with Censoring of Cognitive Radios in Rayleigh Fading Channel," Wireless Personal Communications (WPC), Springer, vol. 70, no. 4, pp. 1409-1424, June 2013.

[22] Sanjay Dhar Roy, S Reddy Vamshidhar Reddy, "Signal Strength Ratio Based Vertical Handoff Decision Algorithms in Integrated Heterogeneous Networks," Wireless Personal Coummunication, Springer (US), Feb. 2014,DOI 10.1007/s11277- 014-1655-9.

[23] Abhijit Bhowmic, Srinivas Nallagonda, Sanjay Dhar Roy,Sumit Kundu,"Cooperative Spectrum Sensing with Double Threshold and Censoring in Rayleigh Faded Cognitive Radio Network," Wireless Personal Coummunication, May. 2015, ISSN 0929-6212, DOI 10.1007/s11277-015-2606-9.

[24] J Ghosh, S Dhar Roy," Qualitative analysis for coverage probability and energy efficiency in cognitive-femtocell networks under macrocell infrastructure," ELECTRONICS LETTERS, 20th August 2015, Vol. 51, No. 17, pp. 13781380 . 